Pacific

Journal of

Mathematics

ANISOTROPIC DIFFUSION ON TOTALLY DISCONNECTED ABELIAN GROUPS

Mauro Del Muto and Alessandro FigÀ-Talamanca

Volume $225 \quad$ No. 2

June 2006 


\title{
ANISOTROPIC DIFFUSION ON TOTALLY DISCONNECTED ABELIAN GROUPS
}

\author{
Mauro Del Muto And Alessandro FigÀ-TAlamancA
}

\begin{abstract}
We consider a locally compact, noncompact, totally disconnected, nondiscrete, metrizable abelian group $G$ that is the union of a countable chain of compact subgroups. On $\boldsymbol{G}$ we consider a stationary standard Markov process defined by a semigroup $\mu_{t}$ of probability measures, satisfying $\mu_{s+t}=$ $\mu_{s} * \mu_{t}$ and $\lim _{t \rightarrow 0} \mu_{t}=\delta_{0}$, and we consider the Lévy measure associated to the process through the Lévy-Khintchine formula. Under the hypothesis that the Lévy measure is unbounded, we show that the process may be obtained as a limit of discrete processes defined on the discrete quotient groups $G / G_{n}$, where $G_{n}$ is a descending chain of compact open subgroups. These discrete processes, in turn, are defined by means of a random walk on a homogeneous tree, naturally associated to $G$.
\end{abstract}

\section{Introduction and preliminaries}

Let $G$ be a locally compact, noncompact, totally disconnected abelian group. We assume that $G$ is metrizable, nondiscrete and the union of a countable chain of compact subgroups. The hypotheses imply that there exists a double sequence of compact open subgroups $\left\{G_{n}: n \in \mathbb{Z}\right\}$, satisfying the following conditions (see [Pontryagin 1966; Evans 1989]):

(i) $G_{n} \subset G_{n-1}$.

(ii) $\bigcup_{n} G_{n}=G$ and $\bigcap_{n} G_{n}=\{0\}$.

(iii) $G_{n} / G_{n+1}$ is a finite group of order $q_{n}$.

Property (iii) implies that $m\left(G_{n}\right)=m\left(G_{n+1}\right) q_{n}$ if $m$ is a Haar measure for $G$. Thus, if we choose the Haar measure in such a way that $m\left(G_{0}\right)=1$, we conclude that $m\left(G_{n}\right)=q_{n} \ldots q_{-1}$ for $n<0$ and $m\left(G_{n}\right)=q_{n-1}^{-1} \ldots q_{0}^{-1}$ for $n>0$.

There is a natural ultrametric structure associated to the double chain of subgroups $G_{n}$. We define a norm for the elements of $G$ :

Definition 1. Define $|0|=0$ and $|a|=m\left(G_{n}\right)$ for $a \in G_{n} \backslash G_{n+1}$.

MSC2000: primary 43A70; secondary 60J60.

Keywords: tree, ultrametric space, totally disconnected group, diffusion, stationary Markov process. 
This norm satisfies the ultrametric inequality

$$
|a+b| \leq \max \{|a|,|b|\} .
$$

It is also clear that $|a|=|-a|$ and that $d(a, b)=|a-b|$ is a metric that gives $G$ the structure of a locally compact ultrametric space satisfying the following properties:

(a) Every closed ball is compact.

(b) The group of isometries acts transitively on the space.

These hypotheses imply, as shown in [Hewitt and Ross 1963; Del Muto and Figà-Talamanca 2004], that the ultrametric space may be given the structure of a locally compact abelian group. The same group structure can be given different (though topologically equivalent) ultrametric structures, through a different choice of the double sequence $G_{n}$. In particular, one could choose a maximal chain of subgroups. In this case the groups $G_{n} / G_{n+1}$ would be simple and the $q_{n}$ would be prime numbers. This choice however may not be always the most appropriate, in particular when $G$ is a local field, because in this case the chain of subgroups which yields the additional property that $|a b|=|a||b|$ may not be maximal.

Conversely, two different group structures may yield the same ultrametric structure. For instance, there exist local fields of characteristic zero and of characteristic $p \neq 0$ that have the same metric structure.

In this paper we assume given the group structure and the chain $G_{n}$ of compact open subgroups of $G$ satisfying the properties listed, and we define the metric structure as above.

We recall from [Del Muto and Figà-Talamanca 2004] that to an ultrametric space such as $G$ we can associate, in a standard fashion, a tree $\mathscr{T}$. The vertices of the tree are the metric balls, and hence in this case the cosets $\left\{G_{n}+a: a \in G, n \in \mathbb{Z}\right\}$. The ascending sequence of subgroups $\left\{G_{n}: n=-1,-2, \ldots\right\}$ identifies a special boundary point, which we denote by $\infty$. With respect to this special point we consider the horocycles of the tree. A horocycle in this case is the set of vertices consisting of the balls of a given radius; in other words the cosets relative to the same subgroup $G_{n}$. Thus, for fixed $n$, a horocycle is the set $\mathfrak{H}_{n}=\left\{G_{n}+a: a \in G\right\}$. The boundary of $\mathscr{T}$ may be identified with the one-point compactification $G \cup\{\infty\}$ of $G$. We refer to [Del Muto and Figà-Talamanca 2004] and [Figà-Talamanca 1997] for a complete treatment of the association between an ultrametric space and the tree of its metric balls. The most complete source for the basic definitions related to the geometry of trees is still [Cartier 1972].

Let $\Gamma$ be the dual group of $G$. Then $\Gamma$ is the union of compact open subgroups $\Gamma_{n}=\left\{\chi \in \Gamma: \chi(a)=1\right.$ for $\left.a \in G_{-n}\right\}$. The order of the quotients $\Gamma_{n} / \Gamma_{n+1}$ is the number $q_{-n}$. Thus $\Gamma$ may be given the structure of an ultrametric space with the same properties as the group $G$. 
Now suppose that $\mu_{t}$ is a semigroup of probability measures on $G$ associated to a standard stationary Markov process on $G$. Then it follows from [Parthasarathy 1967; Kochubei 2001] that there exists a positive measure $F$ on $G$, such that $F\left({ }^{c} G_{n}\right)<\infty$ for every $n$ and

$$
\hat{\mu}_{t}(\chi)=\exp \left(-t \int_{G}(1-\chi(a)) d F(a)\right)
$$

for every $\chi \in \Gamma$. The measure $F$ is called the Lévy measure and the formula (1) is called the Lévy-Khintchine formula. Observe that every character $\chi$ of $G$ takes the value 1 on a neighborhood $G_{n}$ of 0 . Therefore the integral in (1) is always finite.

\section{Discrete approximating processes}

We shall assume henceforth that $F$ is infinite on $G$; in other words, that the sequence $\alpha_{n}=F\left({ }^{c} G_{n}\right)$ is unbounded as $n \rightarrow+\infty$. Observe that $\alpha_{n} \leq \alpha_{n+1}$. Therefore $\lim _{n \rightarrow+\infty} \alpha_{n}=+\infty$. It is also clear that $\lim _{n \rightarrow+\infty} \alpha_{-n}=0$. We will presently show that the process associated with $\mu_{t}$ may be approximated by appropriate convolution powers of certain discrete processes.

Lemma 2. Let $\mu_{t}$ be a semigroup of probability measures associated to a stationary standard Markov process on the group $G$. Let $F$ be the Lévy measure, satisfying, with reference to the semigroup $\mu_{t}$, the Lévy-Khintchine formula (1). Let $\alpha_{n}=F\left({ }^{c} G_{n}\right)$ and suppose that $\lim _{n \rightarrow \infty} \alpha_{n}=\infty$. Let $\lambda_{n}$ be a sequence of measures such that $\lambda_{n}\left(G_{n}\right)=0$ and

$$
\lambda_{n}\left(G_{n}+a\right)=\frac{F\left(G_{n}+a\right)}{\alpha_{n}} \quad \text { if } a \notin G_{n} .
$$

Then

$$
\lim _{n \rightarrow \infty} \lambda_{n}^{*\left\lfloor t \alpha_{n}\right\rfloor}=\mu_{t}
$$

in measure, where $\left\lfloor t \alpha_{n}\right\rfloor$ denotes the integral part of $t \alpha_{n}$.

Proof. We know $\lambda_{n}$ is a probability measure on $G$ because

$$
\frac{1}{\alpha_{n}} \sum_{a \notin G_{n}} F\left(G_{n}+a\right)=1 .
$$

We now compute the Fourier-Stieltjes transform of $\lambda_{n}$ by noticing that if $\chi \in \Gamma_{-n}$, the annihilator of $G_{n}$, we have

$$
\hat{\lambda}_{n}(\chi)=\frac{1}{\alpha_{n}} \int_{{ }^{c} G_{n}} \chi(a) d F(a)=1+\frac{1}{\alpha_{n}} \int_{{ }^{c} G_{n}}(\chi(a)-1) d F(a) .
$$


Since $\Gamma_{-n} \rightarrow \Gamma$ for $n \rightarrow \infty$, and since every character is 1 on a neighborhood of 0 , it follows that, for every $\chi \in \Gamma$,

$$
\lim _{n \rightarrow \infty} \int_{{ }^{c} G_{n}}(\chi(a)-1) d F(a)=\int_{G}(\chi(a)-1) d F(a) .
$$

We conclude that

$$
\lim _{n \rightarrow+\infty} \hat{\lambda}_{n}(\chi)^{\left\lfloor t \alpha_{n}\right\rfloor}=\exp \left(t \int_{G}(\chi(a)-1) d F(a)\right)=\hat{\mu}_{t}(\chi) .
$$

The assumptions on the sequence $\lambda_{n}$ in Lemma 2 are sufficient to characterize it uniquely, under the condition of invariance under translations by elements of $G_{n}$. Such a $G_{n}$-invariant sequence $\lambda_{n}$ may be thought of as a measure on the quotient $G / G_{n}$. Therefore its convolution powers define a process on the horocycle $\mathfrak{H}_{n}=G / G_{n}$. It goes without saying that any measure satisfying the conditions of Lemma 2 can be made $G_{n}$-invariant without losing these properties.

\section{The process on the vertices of the tree}

The geometric structure of an ultrametric space such as $G$ allows for an intuitive description of what a diffusion process should be. Let's consider first of all a discretization of the space, such as $G / G_{n}$. The elements of this space are the metric balls of radius (or diameter) $m\left(G_{n}\right)$, a number which is small when $n$ is large. Consider a particle that has moved out of $G_{n}$, and therefore finds itself in $G_{n-1}$. How could it reach a ball $G_{n}+a$ ? It must obviously move up to larger and larger balls, until it reaches a ball $G_{h}$ large enough to contain $a$. It will then move down on a chain of descending balls until it reaches $G_{n}+a$. It seems natural therefore to associate to a diffusion process on an ultrametric space a random walk on the tree of its metric balls. The transition probabilities should account for the relative difficulty of moving up (or exiting) from a ball to a larger ball, and of moving down (or entering) from a ball to a smaller ball. This idea was put to work in [Figà-Talamanca 1994; 2001; Baldi et al. 2001; Del Muto and Figà-Talamanca 2004]. In this context, the hitting distribution of the process on the metric balls, starting at vertex $G_{n-1}$, defines the measures $\lambda_{n}$, the powers of which approximate $\mu_{t}$ in accordance with Lemma 2.

The chief novelty of the present paper is that we do not assume that the measures $\mu_{t}$ depend only on the distance from 0 . In other words, the diffusion process defined by $\mu_{t}$ is anisotropic (which accounts for the title of this paper). For a different approach to diffusion on ultrametric spaces (in fact on local fields) one should consult the papers of S. Albeverio and his collaborators cited in [Del Muto and Figà-Talamanca 2004]. A full set of references may be found in [Albeverio and Zhao 2000]. 
We now proceed to define the random process on the tree of metric balls of $G$. We introduce first, as in [Del Muto and Figà-Talamanca 2004], the sequence $\zeta_{i}=\alpha_{i} / \alpha_{i+1}$, and we observe that $\lim _{n \rightarrow-\infty} \alpha_{n}=0$ implies that

$$
\lim _{n \rightarrow+\infty} \zeta_{h} \zeta_{h-1} \ldots \zeta_{h-n}=\frac{\alpha_{h-n}}{\alpha_{h+1}}=0 \quad \text { for every } h \in \mathbb{Z} .
$$

We also introduce a sequence of subtrees $\tau_{i}$ of the tree $\mathscr{T}$. For $i \in \mathbb{Z}$, the vertices of $\tau_{i}$ are $G_{i}$ and the metric balls (cosets) contained in $G_{i} \backslash G_{i+1}$. The sets of vertices of the trees $\tau_{i}$ are disjoint and their union is the set of vertices of $\mathscr{T}$. We use, with a slight abuse of language, the same notation $\tau_{i}$ for the tree and its set of vertices. We use the notation $x \sim y$ to denote two adjacent vertices.

We now define, for $x$ and $y$ two metric balls (cosets), the probability $P(x, y)$ of going from $x$ to $y$ in one step. First we stipulate that $P(x, y)=0$ if $x$ is not adjacent to $y$. We assume that $x \in \tau_{i}$ and define $P(x, y)=0$ if $x=G_{i}$ and $y=G_{i+1}$. This means that according to the random walk $P$ it is not possible to go down on the geodesic $\left\{G_{i}\right\}$. Finally, if $x \in \tau_{i}$ and $x \sim y \neq G_{i+1}$, we set

$$
P(x, y)= \begin{cases}\frac{\zeta_{i}}{1+\zeta_{i}} & \text { if } x \subset y, \\ \frac{1}{1+\zeta_{i}} \frac{F(y)}{F(x)} & \text { if } y \subset x \text { and } x \neq G_{i}, \\ \frac{1}{1+\zeta_{i}} \frac{F(y)}{F\left(G_{i} \backslash G_{i+1}\right)} & \text { if } x=G_{i}, y \subset x \text { and } y \neq G_{i+1}\end{cases}
$$

This definition does not cover the case in which the denominators of the quotients $F(y) / F\left(G_{i} \backslash G_{i+1}\right)$ or $F(y) / F(x)$ are zero. To complete the definition we stipulate that the quotient $F(y) / F\left(G_{i} \backslash G_{i+1}\right)$ is replaced by $m(y) / m\left(G_{i} \backslash G_{i+1}\right)$ if $F\left(G_{i} \backslash G_{i+1}\right)=0$, and $F(y) / F(x)$ is replaced by $m(y) / m(x)$ if $F(x)=0$. As a result the probability of going up from the vertex $x$ to the vertex immediately above in one step is always $\zeta_{i} /\left(1+\zeta_{i}\right)$.

We observe that $P(x, y)$ is defined for every pair of vertices because $\mathscr{T}$ is the disjoint union of the subtrees $\tau_{i}$.

For fixed $i$ we consider the probability $\eta_{i k}$ that, starting at a vertex of $\tau_{i}$ that belongs to a horocycle $\mathfrak{H}_{k}$ (with $k \geq i$ ), we reach sooner or later a vertex of the horocycle $\mathfrak{H}_{k-1}$. We can prove, as in [Del Muto and Figà-Talamanca 2004], that this probability is independent of $k$ and equals $\zeta_{i}$. We recall here the proof for convenience. Every path of the process $P$ that starts at a vertex of $\tau_{i}$ and moves outside $\tau_{i}$ visits the horocycle $\mathfrak{H}_{k-1}$ before going outside $\tau_{i}$ (if $k>i$ ) or as its first step outside $\tau_{i}$ (if $k=i$ ). Thus the probability of visiting $\mathfrak{H}_{k-1}$ given that one starts at a vertex of $\tau_{i}$ belonging to $\mathfrak{H}_{k}$ remains the same if this event is conditioned to the event that the process $P$ remains within the subtree $\tau_{i}$ before reaching $\mathfrak{H}_{k-1}$. 
But within the subtree,

$$
\eta_{i k}=\frac{\zeta_{i}}{1+\zeta_{i}}+\frac{1}{1+\zeta_{i}} \eta_{i k}^{2}
$$

This implies that $\eta_{i k}=\zeta_{i}$ is independent of $k$. We have proved in particular that $\zeta_{i}$ is the probability of visiting the horocycle $\mathfrak{H}_{i-1}$ starting from $G_{i}$.

Lemma 3. Let $n$ be any integer and $\mathfrak{H}_{n}$ the corresponding horocycle. Starting at any vertex of the tree $\mathcal{T}$, with probability one, the random walk defined by $P$ keeps, after a finite number of steps, below the horocycle $\mathscr{H}_{n}$.

Proof. We show first that, with probability one, after a finite number of steps, the random walk will stay inside one of the subtrees $\tau_{i}$. This will certainly be the case if the random walk never visits a vertex of the double geodesic $\left\{G_{k}: k \in \mathbb{Z}\right\}$. Suppose then that a vertex $G_{h}$ is visited. We observed above that

$$
\lim _{n} \zeta_{h} \zeta_{h-1} \ldots \zeta_{h-n}=0
$$

for every fixed integer $h$. But the product $\zeta_{h} \zeta_{h-1} \ldots \zeta_{h-n}$ is the probability that starting at the vertex $G_{h}$, we reach sooner or later the vertex $G_{h-n}$. We conclude that the probability that the random walk defined by $P$ will move up indefinitely on the geodesic $\left\{G_{k}: k \in \mathbb{Z}\right\}$ is zero. This means that, with probability one, the random walk will stay indefinitely inside one of the subtrees $\tau_{i}$ (corresponding to the $G_{i}$ of lowest index ever reached). The fact that the random walk visits and stays inside $\tau_{i}$ implies that $\zeta_{i}<1$, because the case $\zeta_{i}=1$ implies that within $\tau_{i}$ the probability of going up is the same as the probability of going down, which in turn implies that the random walk cannot keep indefinitely inside $\tau_{i}$. The condition $\zeta_{i}<1$ implies that $\zeta_{i} /\left(1+\zeta_{i}\right)<\frac{1}{2}$. This is the probability of moving within $\tau_{i}$ from a horocycle $\mathfrak{H}_{k}$ to a horocycle $\mathfrak{H}_{k-1}$ in one step. We conclude that it is more probable to go down to the horocycle of larger index and that the random walk eventually stays below the horocycle $\mathfrak{H}_{n}$.

Of course, the vertices that lie below the horocycle $\mathfrak{H}_{n}$ are the balls strictly contained in the balls of $\mathfrak{H}_{n}$. Abusing language slightly we may say that, starting at any vertex, the random walk eventually stays inside a ball of arbitrarily small radius.

We now fix an integer $n$ and, for $x \in \mathfrak{H}_{n}$, we define $\lambda_{n}(x)$ to be the probability that the random walk, starting at the vertex $G_{n-1}$, visits and stays indefinitely below (or inside) the vertex $x$. The probability measure $\lambda_{n}$ may also be thought of as the conditional expectation with respect to the partition $\mathfrak{H}_{n}=G / G_{n}$ of the hitting distribution of $P$, starting at the vertex $G_{n-1}$, on the boundary of the tree.

Lemma 4. The measures $\lambda_{n}$ satisfy the hypothesis of Lemma 2. 
Proof. Under the assumption that the random walk $P(x, y)$ starts at $G_{n-1}$ we define $\psi(x)$ to be the probability that the random walk visit $x$ and keeps eventually below the vertex (inside the ball) $x$. The function $\psi$ is defined for every vertex of the tree, although it is zero for infinitely many vertices, and does not sum to one. By definition $\psi\left(G_{n}\right)=0$, and $\psi(x)=\lambda_{n}(x)$, for $x \in \mathfrak{H}_{n}$. Let $x \in \mathfrak{H}_{n}$ and suppose $x \neq G_{n}$. Let $\left\{x=x_{0}, x_{1}, \ldots x_{h}=G_{n-h}\right\}$ be the chain of vertices connecting $x$ to the geodesic $\left\{G_{i}\right\}$. Then

$$
\lambda_{n}(x)=\psi\left(x_{1}\right) \frac{F(x)}{F\left(x_{1}\right)} .
$$

Indeed if we assume that the random walk stays eventually inside $x_{1}$ we may conclude that there is a last time in which $x_{1}$ is visited. At that time the probability $F(x) / F\left(x_{1}\right)$ that $x$ is visited at the following step equals the probability of staying eventually inside $x$. Iterating this reasoning we conclude that

$$
\begin{aligned}
\lambda_{n}(x) & =\psi\left(x_{2}\right) \frac{F(x)}{F\left(x_{1}\right)} \frac{F\left(x_{1}\right)}{F\left(x_{2}\right)}=\cdots=\psi\left(G_{n-h}\right) \frac{F(x)}{F\left(G_{n-h} \backslash G_{n-h+1}\right)} \\
& =\zeta_{n-1} \ldots \zeta_{n-(h-1)}\left(1-\zeta_{n-h}\right) \frac{F(x)}{F\left(G_{n-h} \backslash G_{n-h+1}\right)} \\
& =\frac{\alpha_{n-1}}{\alpha_{n}} \frac{\alpha_{n-2}}{\alpha_{n-1}} \ldots \frac{\alpha_{n-h+1}-\alpha_{n-h}}{\alpha_{n-h+1}} \frac{F(x)}{\alpha_{n-h+1}-\alpha_{n-h}}=\frac{F(x)}{\alpha_{n}},
\end{aligned}
$$

showing that $\lambda_{n}$ satisfies the hypothesis of Lemma 2.

We now have all the ingredients needed to make our construction. Using the Lévy measure associated to the process $\mu_{t}$ we define the process $P(x, y)$ on the vertices of the tree $\mathscr{T}$ of $G$, which accounts for the relative difficulty of exiting or entering a ball. We consider the hitting distribution on the boundary $G$ of the tree, of the process $P(x, y)$ starting at the vertex $G_{n-1}$. The conditional expectations with respect to the partition provided by $\mathfrak{H}_{n}$ of these hitting distributions are measures $\lambda_{n}$ that define discrete processes on the horocycles $\mathfrak{H}_{n}$. Appropriate powers of these measures approximate the measures $\mu_{t}$.

\section{Stable processes}

These results apply in particular to processes associated to a stable distribution on a local field. When $G$ is a locally compact field it is convenient to choose the sequence $G_{n}$ in such a way that the norm associated to the sequence satisfies $|a b|=|a||b|$. This is achieved by letting $G_{0}$ be the $\operatorname{ring}$ of integers of $G$ and $G_{1}$ its unique maximal ideal. Next, one chooses a generator $\mathfrak{p}$ of the maximal ideal $G_{1}$, and defines $G_{n}=\mathfrak{p}^{n} G_{0}$. The quotient $G_{0} / G_{1}$ is a finite field of order $q=p^{h}$, which is also the order of each quotient $G_{n} / G_{n+1}$. In this context, the most general definition of a stable distribution was the one given by A. N. Kochubei [1998], who 
showed that stable processes satisfying the additional condition $\mu_{t}(E)=\mu_{t}(-E)$ for any Borel set $E$ are associated to Lévy measures having the following property: For some $\alpha>0$, there exists a positive integer $N$ and a generator $\mathfrak{p}$ of the maximal ideal in the ring of integers, such that

$$
F\left(\mathfrak{p}^{N} B\right)=q^{N \alpha} F(B) \quad \text { for every ball } B \in G .
$$

Conversely, if the Lévy measure enjoys this property, the associated process is stable. The preceding equation implies, for the sequence $\left\{\zeta_{n}\right\}$,

$$
\zeta_{n} \cdots \zeta_{n+N-1}=q^{N \alpha}
$$

This condition holds if and only if the double sequence $\left\{\zeta_{n}: n \in \mathbb{Z}\right\}$ is periodic. The simplest (isotropic) case occurs when $N=1, \zeta_{n}=\zeta$ is constant, and $F$ is uniformly distributed on $G_{n} \backslash G_{n+1}$. This was the case studied in [Baldi et al. 2001], which yielded $\zeta=q^{-\alpha}$ and

$$
\hat{\mu}_{t}(\xi)=e^{-t|\xi|^{\alpha}}
$$

\section{References}

[Albeverio and Zhao 2000] S. Albeverio and X. Zhao, "On the relation between different constructions of random walks on p-adics”, Markov Process. Related Fields 6:2 (2000), 239-255. MR 2001i:60007 Zbl 0974.60028

[Baldi et al. 2001] P. Baldi, E. Casadio Tarabusi, and A. Figà-Talamanca, "Stable laws arising from hitting distributions of processes on homogeneous trees and the hyperbolic half-plane", Pacific J. Math. 197:2 (2001), 257-273. MR 2001m:60015 Zbl 1049.60015

[Cartier 1972] P. Cartier, "Fonctions harmoniques sur un arbre", pp. 203-270 in Symposia Mathematica (Rome, 1971), vol. IX, Academic Press, London, 1972. MR 50 \#5950 Zbl 0283.31005

[Del Muto and Figà-Talamanca 2004] M. Del Muto and A. Figà-Talamanca, "Diffusion on locally compact ultrametric spaces”, Expo. Math. 22 (2004), 197-211. MR 2005c:60004 Zbl 02222514

[Evans 1989] S. N. Evans, "Local properties of Lévy processes on a totally disconnected group", J. Theoret. Probab. 2:2 (1989), 209-259. MR 90g:60069 Zbl 0683.60010

[Figà-Talamanca 1994] A. Figà-Talamanca, "Diffusion on compact ultrametric spaces", pp. 157167 in Noncompact Lie groups and some of their applications (San Antonio, TX, 1993), edited by E. A. Tanner and R. Wilson, NATO Adv. Sci. Inst. Ser. C Math. Phys. Sci. 429, Kluwer Acad. Publ., Dordrecht, 1994. MR 95m:60012 Zbl 0839.43011

[Figà-Talamanca 1997] A. Figà-Talamanca, "Local fields and trees", pp. 3-16 in Harmonic functions on trees and buildings (New York, 1995), edited by A. Korányi, Contemp. Math. 206, Amer. Math. Soc., Providence, RI, 1997. MR 98h:22005 Zbl 0892.22003

[Figà-Talamanca 2001] A. Figà-Talamanca, "An application of Gelfand pairs to a problem of diffusion in compact ultrametric spaces", pp. 51-67 in Topics in probability and Lie groups: boundary theory, edited by J. C. Taylor, CRM Proc. Lecture Notes 28, Amer. Math. Soc., Providence, RI, 2001. MR 2002h:60008 Zbl 0985.60004

[Hewitt and Ross 1963] E. Hewitt and K. A. Ross, Abstract harmonic analysis, I: Structure of topological groups, integration theory, group representations, Grundlehren der math. Wiss. 115, Springer, Berlin, 1963. MR MR0156915 (28 \#158) Zbl 0115.10603 
[Kochubei 1998] A. N. Kochubei, "Limit theorems for sums of $p$-adic random variables", Exposition. Math. 16:5 (1998), 425-439. MR 99m:60016 Zbl 0954.60001

[Kochubei 2001] A. N. Kochubei, Pseudo-differential equations and stochastics over non-archimedean fields, Pure and App. Math. 244, Marcel Dekker, New York, 2001. MR 2003b:35220 Zbl 0984.11063

[Parthasarathy 1967] K. R. Parthasarathy, Probability measures on metric spaces, Prob. Math. Stat. 3, Academic Press, New York, 1967. MR 37 \#2271 Zbl 0153.19101

[Pontryagin 1966] L. S. Pontryagin, Topological groups, Gordon and Breach, New York, 1966. MR 34 \#1439 Zbl 0882.01025

Received October 2, 2004.

Mauro Del Muto

ACE S.N.C.

Via Aulo Plauzio 6

00181, ROMA

ITALY

mauro.delmuto@acesys.it

Alessandro FigÀ-TAlamanca

DIPARTIMENTO DI MATEMATICA

UNIVERSITÀ DEGLI STUdi DI ROMA "LA SAPIENZA"

PiAzZAle Aldo Moro 5

00185, ROMA

ITALY

sandroft@mat.uniroma1.it 